\title{
Rice mutants with tolerance to multiple abiotic stresses show high constitutive abundance of stress-related transcripts and proteins
}

\author{
Priyanka Das ${ }^{1}$, Rajeev N Bahuguna ${ }^{1}$, Ray Singh Rathore ${ }^{1}$, Sheenu Abbat ${ }^{1}$, Ramsong C. \\ Nongpiur ${ }^{1}$, Fatma Sarsu ${ }^{2}$, Sneh L. Singla-Pareek ${ }^{3}$ and Ashwani Pareek ${ }^{1 *}$
}

\author{
${ }^{1}$ Stress Physiology and Molecular Biology Laboratory, School of Life Sciences, Jawaharlal \\ Nehru University, New Delhi 110067, India \\ ${ }^{2}$ Plant Breeding and Genetics Section, Joint FAO/IAEA Division of Nuclear Techniques in \\ Food and Agriculture, Vienna, Austria \\ ${ }^{3}$ Plant Stress Biology, International Centre for Genetic Engineering and Biotechnology, Aruna \\ Asaf Ali Marg, New Delhi 110067, India
}

\section{*Corresponding author: ashwanip@mail.jnu.ac.in}

\begin{abstract}
Mutation breeding has a long track record in the development of crop cultivars with improved tolerance to abiotic stresses such as heat, salinity and drought. Oryza sativa L. cv IR64 is a very popular high yielding rice, but susceptible to major abiotic stresses, such as low and high temperatures, salinity and drought. We subjected IR64 to gamma irradiation and generated a mutant population $\left(\mathrm{M}_{3}\right)$ with $\sim 2,000$ families. These were screened at the seedling stage for tolerance to high-temperature stress using hydroponics and controlled-environment chambers, resulting in the identification of three mutant lines showing a robust seedling phenotype. Under heat stress, higher $\mathrm{CO}_{2}$ assimilation (10-30\%), higher spikelet fertility (40-45\%) and higher antioxidant activity (15-20\% catalase activity) confirmed superiority of the selected mutant lines over wild type plants at seedling and flowering stages. Upon exposure to salinity and drought stress, the three selected lines also exhibited better tolerance than wild type in terms of higher $\mathrm{CO}_{2}$ assimilation, stomatal conductance, transpiration and chlorophyll fluorescence. Transcript and protein abundance analyses confirmed higher constitutive levels of heat shock proteins and antioxidant enzymes in the mutant lines relative to wild type. Tolerance to multiple abiotic stresses was reflected in higher (25-30\%) grain yield than wild type. It is anticipated that the mutant lines identified will be useful for developing new improved cultivars for dry and saline areas and may be exploited to dissect the molecular basis of multiple stress tolerance in crop plants.
\end{abstract}

Key Words: Rice, Mutant, Gamma irradiation, heat, salinity, drought.

Abbreviations: CAT_Catalase, APX_Ascorbate Peroxidase, SOD_Superoxide Dismutase, GLY_Glyoxalase, HSP_Heat Shock Protein.

\section{Introduction}

Rice is a staple food crop especially in Asia and Africa. Despite growing demands to feed an ever-increasing population, rice production has been decreasing at a rate of $2.7 \%$ per year in $1970-1990$ to $1.2 \%$ per year in 1990-2007 (Wassman et al., 2009). Sensitivity of rice to abiotic stresses such as heat, drought and salinity pose major constraints to yield (Grover and Minhas, 2000). 'Abiotic stress tolerance' and 'stability in yield' are complex genetic traits which involve highly regulated expression of a plethora of genes or the indirect effect of a single key regulatory gene (Yokoi et al., 2002). Hence, it is a daunting task for rice researchers to adopt a suitable method/technique to tailor abiotic-stress tolerance with improved yield and yield stability under unfavourable environmental conditions.

Mutation breeding has made notable contributions towards the production of high-yielding and stress-tolerant crops (Cassell and Doyel, 2003; Parry et al., 2009; Bado et al., 2015). There are now simple and effective protocols available to screen for salt-tolerant rice mutants (Badoet al., 2016a; Joshi et al., 2016). Rice seeds irradiated with $\mathrm{C}$ or $\mathrm{Ne}$ ions have successfully generated mutant genotypes with tolerance to salinity (Hayashi et al., 2007). Moreover, the mutant rice genotype, Zhefu 802, induced by gamma radiation in the parental cultivar Simei no. 2, showed cold tolerance (Ahloowalia et al., 2003). Many other rice genotypes such as, A-20, Atomita 2, Changwei19, Emai No. 9, Fuxuan No. 1, Liaoyan 2, Mohan-CSR 4, Jiaxuan No. 1, Shua 92, Nipponbare, Basmati 370, IR 58614, Zhefu 802, Kasmir basmati and mutated
IR8 have been generated through mutation breeding for improving tolerance against various abiotic stresses (Singh, 2000; Baloch et al., 2003; Ahloowalia et al., 2003; Chhun et al., 2003; Saleem et al., 2005; Hayashi et al., 2008; Jain and Suprasanna, 2011). Cultivars have also been developed from these lines and released all over the world.

Heat stress has a dynamic impact on productivity of crop plants where sensitivity varies with the development stage and tissue type (Barnabas et al., 2008; Sakata and Higashitani, 2008). Heat stress at the seedling stage or flowering stage or grain filling stage can have a significant impact on plant survival and grain yield (Bahuguna et al., 2015).

Most reports on crop varieties tolerant to abiotic stress have centred on single stresses, and experiments have been usually conducted under controlled environmental conditions. However, under field conditions, crops are seldom exposed to a single stress; rather they are exposed to more than one type of stress either sequentially or simultaneously. Therefore, improving crops for tolerance towards multiple stresses is an imperative. Although, efforts need to focus on integrating tolerance to multiple abiotic stresses, reports in rice are scant. Most successful breeding programmes have been directed towards improving tolerance to a distinct stress, such as drought, salinity, flooding or high temperature. 
In this study we generate and screened $\sim 2,000 \quad \mathrm{M}_{3}$ families derived from gamma-irradiated rice (cv IR64) for heat tolerance at the seedling stage (by evaluating phenotypic, physiological and biochemical parameters). Subsequently, three selected putative heat-tolerant mutant lines were screened for their tolerance at flowering and grain filling stages to multiple abiotic stresses such as heat, salinity and drought.

\section{Results}

\section{Heat stress at the seedling stage}

Exposure of rice wild type (WT) seedlings to heat stress affected the growth of shoot and roots relative to control seedlings without stress (Fig1a). Under control conditions with no heat stress, no difference in seedling shoot length was observed between WT and the selected mutant families (D100/79, D100/96 and D100/11). This was not the case for high temperature stress (Fig1b). Under heat stress conditions, mutant lines exhibited a higher shoot length ranging from a $25 \%$ increase in $\mathrm{D} 100 / 79$ to $39 \%$ increase in D100/96 as compared to WT (Fig1b). Comparisons of root length revealed that even under control conditions, mutant seedlings had longer roots as compared to the WT (Fig1c). In the mutant families D100/79, D100/111 and D100/96, roots were $17 \%, 59 \%$ and $22 \%$ longer than WT under control conditions. A similar phenotype was observed under heat stress conditions, where roots of D100/79, D100/111 and D100/96 were $30 \%, 48 \%$ and $30 \%$ longer compared to W'T, respectively (Fig1c). For fresh weight, a similar observation was made, where the mutant families had higher weights than WT seedlings under both control and heat stress conditions (Fig1d). Under control conditions, the fresh weight of D100/79, D100/111 and D100/96 seedlings were 9\%, 25\% and $13 \%$ higher than WT, respectively. Further, under heat stress conditions, the fresh weight of D100/79, D100/111 and D100/96 seedlings were $22 \%, 25 \%$ and $23 \%$ higher relative to WT, respectively (Fig1d).

To understand the basis of improved seedling phenotypes, we carried out heat stress associated physiological and biochemical analyses such as membrane stability, activity of antioxidant enzymes and chlorophyll content in the seedlings (Lakra et al., 2017). Under control condition without stress, significant differences in electrolyte leakage were observed between WT and the selected mutant families where the mutants showed decreased electrolyte leakage compared to WT (Fig2a). The electrolyte leakage in D100/79, D100/111 and D100/96 seedlings were $4 \%, 6 \%$ and $9 \%$ lower than WT, respectively. Heat stress treatment significantly increased electrolyte leakage across the WT and mutant plants, but the mutant families recorded significantly lower electrolyte leakage relative to $\mathrm{W} T$, with D100/111 recording the lowest electrolyte leakage $(26 \%$ less than WT; Fig2a). Membrane stability was checked using lipid peroxidation (MDA content) analysis. Under control conditions, no significant difference in lipid peroxidation was observed among WT and mutant seedlings. However, under heat stress, lipid peroxidation was highest for WT plants $\left(\sim 400 \mathrm{nmol} \mathrm{g}^{-1}\right.$ fresh weight). Mutant families recorded $0.5 \%$ to $2 \%$ lower MDA content as compared to WT under similar conditions, although the only significant change as compared to WT was for D100/79 (Fig2b). Interestingly, significant differences in catalase activity of two mutants (D100/79 and D100/96) were observed under control conditions as compared to WT. D100/79 and D100/96 showed $10.8 \%$ and $4 \%$ higher catalase activity than WT controls, respectively.

However, D100/111 seedlings did not show any significant increase in catalase activity in comparison to WT. Interestingly, heat stress led to a significant increase in catalase activity in D100/111 seedlings and similar increase was also observed in D100/96 seedlings, when compared to WT (Fig2c). Quite contrary to the catalase activity, under control conditions, no significant differences were observed between APX activity of WT and the three mutant families (Fig2d).
But after heat stress treatment, D100/79 and D100/111 mutant seedlings showed significantly higher APX activity $(8.2 \%$ and $5.2 \%$ increase) compared to WT. No significant differences were observed in the APX activity of WT and D100/96 heat-stressed seedlings. The total chlorophyll content of WT and mutant seedlings was analysed under heat stress. While no differences were observed for chlorophyll content of WT and mutant seedlings under control conditions, under heat stress two of the mutants viz. D100/79 and D100/111, maintained a chlorophyll content which was respectively $50 \%$ and $16 \%$ higher than WT (Fig2e).

\section{Heat tolerance at reproductive stages}

Since heat stress at reproductive stages has severe effects on yield of rice, the effects of heat stress at the flowering stage on the reproductive development of $\mathrm{WT}$ and mutant plants were analysed (Fig3a). Figure 3b showed panicles of plants under control conditions and after being subjected to heat stress at flowering. Under control conditions, there was no marked difference between spikelet fertility of W'T and mutant plants which ranged from $85.7 \%$ to $89.9 \%$ (Fig $3 \mathrm{c}$ ). Heat stress exposure $\left(40^{\circ} \mathrm{C}\right)$ during the flowering stage led to significant reductions in spikelet fertility of WT and the D100/79 mutant line, recording only $47.7 \%$ and $47.3 \%$ spikelet fertility, respectively (Fig3c). Interestingly, mutant families D100/111 and D100/96 recorded $\sim 80 \%$ spikelet fertility under heat stress which is comparable to that of unstressed, control plants (Fig3c). All the three mutant families recorded a significantly higher number of grains per panicle as compared to WT (Fig3d). Under heat stress, the difference between the number of grains per panicle of WT and mutants was more pronounced, numbers for D100/79, $\mathrm{D} 100 / 111$ and D100/96 were $35.5 \%, 50.0 \%$ and $44.0 \%$ higher than that WT controls (Fig3d). The weight of 1,000 grains from each mutant family was then compared with that of WT. The 1,000-grain weight of each mutant family was significantly higher than W'T under control and stress conditions. 1,000 grain weight differences were $44.6 \%$ and $47.5 \%$ for D100/79, $42.8 \%$ and $43 \%$ for D100/111, $41 \%$ and $36 \%$ for D100/96, as compared to WT under control and heat stress respectively (Fig3e). These analyses showed better performances of the mutant families, not only under heat stress, but also under control conditions.

\section{Gas exchange and chlorophyll fluorescence analyses}

Gas exchange traits and chlorophyll fluorescence data revealed significantly better performance of mutant families as compared to WT under drought (70 to $80 \mathrm{kPa})$, salinity $(200 \mathrm{mM})$ and heat stress $\left(40^{\circ} \mathrm{C}\right)$ at flowering (Fig4a-d) and early grain filling stages (Fig4e-h). Heat and osmotic stress (salinity and drought) treatments significantly reduced $\mathrm{CO}_{2}$ assimilation in the three mutant genotypes as compared to WT controls. WT plants recorded lower $\mathrm{CO}_{2}$ assimilation rates under various stresses as compared to the mutants, being lowest under drought stress viz. 6.9 and $7.7 \mu \mathrm{mol} \mathrm{m} \mathrm{m}^{-2} \mathrm{~s}^{-1}$ at flowering and grain filling stages, respectively (Fig4a and e). However, under heat stress treatment at grain filling, no significant difference was observed in the $\mathrm{CO}_{2}$ assimilation rate of D100/79 plants as compared to WT (Fig4e). All three mutant lines showed significantly higher $\mathrm{CO}_{2}$ assimilation than WT across the osmotic stress treatments, recording 9.5 to $16.1 \mu \mathrm{mol} \mathrm{m} ~_{-2} \mathrm{~s}^{-1}$ photosynthetic activity at flowering and grain filling (Fig4a and e). Further, stomatal conductance reduced significantly across the treatments with WT plants recording the lowest

measurements $\left(0.07\right.$ and $0.1 \mathrm{~mol} \mathrm{H}_{2} \mathrm{O} \mathrm{m}^{-2} \mathrm{~s}^{-1}$, at flowering and grain filling, respectively) under drought stress (Fig4b and f). On the other hand, mutant families recorded relatively higher stomatal conductance under various treatments. D100/96 recorded the highest stomatal conductance after stress treatments at flowering and grain filling stages (Fig4b and f). 
Further, both drought and salinity stress reduced transpiration rate, and WT plants recorded the lowest rates under drought $(2.2$ to $\left.3.3 \mathrm{~mol} \mathrm{H}_{2} \mathrm{O} \mathrm{m}^{-2} \mathrm{~s}^{-1}\right)$ and salinity stress $\left(3.2\right.$ to $4.5 \mathrm{~mol} \mathrm{H}_{2} \mathrm{O} \mathrm{m}^{-}$ ${ }^{2} \mathrm{~s}^{-1}$ ) at both growth stages (Fig4c and g). Conversely, the three mutant families showed higher transpiration rates under drought (3.3 to $7 \mathrm{~mol} \mathrm{H}_{2} \mathrm{O} \mathrm{m}^{-2} \mathrm{~s}^{-1}$ ) and salinity (5.3 to $7.9 \mathrm{~mol} \mathrm{H}_{2} \mathrm{O} \mathrm{m}^{-2} \mathrm{~s}^{-}$ $\left.{ }^{1}\right)$, at flowering and grain filling stages (Fig4c and g). Mutant families recorded higher transpiration rates even under heat stress, with D100/96 showing the highest transpiration rate, 12.4 and $9.1 \mathrm{~mol} \mathrm{H}_{2} \mathrm{O} \mathrm{m}^{-2} \mathrm{~s}^{-1}$ at flowering and grain filling, respectively (Fig4c and 4g). Furthermore, chlorophyll fluorescence reduced significantly across the stress treatments with highest reductions recorded in WT plants at flowering (0.49 to 0.54) and grain filling ( 0.55 to 0.62 ) (Fig 4 dand h). Conversely, mutant lines maintained significantly higher chlorophyll fluorescence ( 0.57 to 0.77 ) over WT plants across the stress treatments and growth stages.

\section{Grain yield under vatious osmotic stresses}

Grain yield was reduced significantly in WT plants and mutants exposed to salinity, drought or heat stress at flowering and grain filling stages (Fig 5). However, the reduction in grain yield was significantly lower in the mutant families compared to WT across stress treatments. Highest reductions in grain yield were recorded for WT plants under drought stress at both flowering (61\%) and grain filling (64\%) stages, compared to control conditions (Fig 5). Yield loss under drought was lower in the mutant lines showing only 52 to $60 \%$ and 48 to $49 \%$ reduction at flowering and grain filling stages, respectively (Fig 5). Similarly, both heat and salinity stress reduced yield in WT plants by 27 to $60 \%$, and heat-induced loss was greater $(39 \%)$ at flowering. However, the mutant families showed significantly lesser reductions in grain yield under both heat and salinity stresses except the line D100/79. Mutant lines D100/111 and D100/96 recorded significantly higher yields under heat and salinity stresses, with D100/111 recording the highest yield under salinity (30, and $48 \mathrm{~g}$ plant $\left.{ }^{-1}\right)$ and heat (48 and $\left.56 \mathrm{~g} \mathrm{plant}^{-1}\right)$ stress at flowering and grain filling, respectively (Fig 5).

\section{Transcript and protein abundance of stress related genes}

Transcript abundance of various key stress-responsive genes have been reported to provide information on regulating osmotic and heat stress tolerance in rice (Kumari et al., 2009; Lakraet al., 2015; Soda et al., 2016). qRT-PCR analyses of histone-binding protein, antioxidant enzymes (APX and SOD) and heat shock protein-70 under heat stress treatment revealed significant differences all genotypes tested (Fig6a). WT plants recorded lowest expression levels of histone binding protein-1b (HBP1b), $A P X, S O D$ and HSP7O. In contrast, all three mutant families displayed significantly higher expression levels of these genes compared to WT, with D100/111 and D100/96 showing considerably higher levels of HBP1b, APX, SOD and HSP-70 activity (Fig6a). Conversely, $C A T$ expression levels in the mutant lines was similar to that observed in WT under heat stress, except in D100/96 which recorded significantly higher level of CAT expression (Fig6a). Thus, the tolerance traits observed in the three mutant families could be attributed to the expression of these positive regulators of stress.

\section{Discussion}

Rice cultivars with multiple abiotic stress tolerance in field conditions are of great elevance (Nguyen and Ferrero, 2006, Das et al., 2014). Mutation breeding provides a promising approach for tailoring rice genotypes for resilience towards environmental stresses (Das et al., 2014; Bado et al., 2015). Although sensitive to several abiotic stresses, IR64 is one of the most popular rice cultivars due to its high-quality grain, high yield and excellent cooking qualities; it is grown in over 200 countries (Laborte et al., 2015, Das et al., 2015b). With an objective of generating new variation in IR64 for resilience to abiotic stresses, we raised a mutant population from gamma-ray mutagenesis and performed screening for high-temperature tolerance. A simple but efficient protocol was developed where screening was done at the seedling stage and further evaluation of selected lines was carried out at flowering and grain filling stages with major emphasis on the phenotypic, physiological, biochemical and molecular parameters.

Rice seeds $\left(\mathrm{M}_{\mathrm{o}}\right)$ were exposed to $100 \mathrm{~Gy}$ of gamma irradiation followed by repeated selfing $\left(\mathrm{M}_{1}\right.$ and $\left.\mathrm{M}_{2}\right)$ to obtain $\mathrm{M}_{3}$ families from which stable lines may be developed. The heat stress treatment and recovery were done based on screening protocols for rice seedlings (Bado et al., 2016a; Joshi et al., 2016). As seedling and reproductive stages are the two most sensitive stages in rice, a strategy was devised to screen the whole population for high temperature tolerance of seedling stages followed by testing selected lines at reproductive stages (flowering and grain filling). We tested three putative mutant lines for tolerance to salinity and drought, in addition to high temperature stress. This strategy allowed us to develop a robust and reproducible pre-field screening method through hydroponics in a short time where the temperature, light and humidity could be controlled very precisely in a plant growth chamber. Plant morphology plays an important role in identifying and selecting mutant lines tolerant to heat or any other stress from among a large population (Ayeneh et al. 2002). Therefore, the first round of screening was based mainly on phenotype, such as leaf tip burn, root length, shoot length, greenness and seedling establishment (Fig1). Previous studies exploring the effects of high temperature on rice seedlings have documented damage of plant cellular architecture, which brings about major changes in chloroplast and mitochondria resulting in reduced metabolism and hence, reduced growth of seedlings under such conditions (Pareek et al., 1997).

Table 1. List of primers used for qRT-PCR and their sequences (5'- $\left.3^{\prime}\right)$.

\begin{tabular}{|llll|}
\hline Hene & Experiment & Forward Primer sequence $(\mathbf{F})$ & Reverse Primer sequence $(\mathbf{R})$ \\
\hline SOD & qRT-PCR & GGATAGCCAACCTTCAGCAG & ATTTGGCCCATGTAGTTTGC \\
\hline CAT & qRT-PCR & CCAGAAGCACCACGCCACCT & GATTGACATGGCCTCCGC \\
\hline APX & qRT-PCR & ATGGATCCCTACAAGCATCGG & AGATGATAGTCCTCAAGGAGG \\
\hline HSP70 & qRT-PCR & CCAGCTAAGAAGCTCCAAG & AGGTGGGGGTGCAGGTTTGTC \\
\hline Actin & qRT-PCR & CAGCCACACTGTCCCCATCTA & AGCAAGGTCGAGACGAAGGA \\
\hline
\end{tabular}



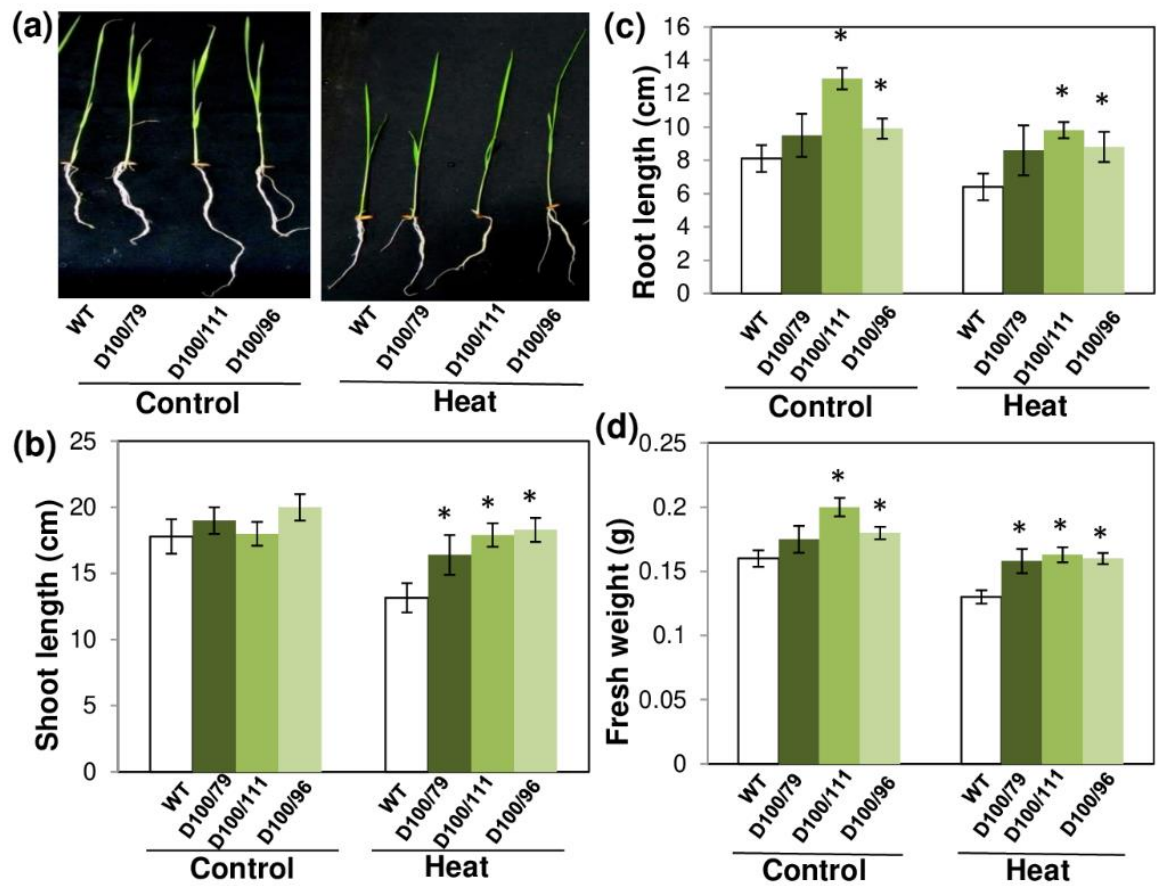

Fig 1. Phenotype analysis of three selected mutant $\left(\mathrm{M}_{3}\right)$ families under control conditions and after heat stress treatment $\left(45^{\circ} \mathrm{C}\right.$ for $\left.12 \mathrm{~h}\right)$. (a) Picture of three selected mutant lines along with the WT under control conditions and after heat stress treatment; Determination of (b) shoot length (c) root length and (d) fresh weight of selected mutant seedlings in comparison to WT under control conditions and after heat stress treatment. Vertical column represents mean of three biological replicates $\pm \mathrm{SE}$. ' $*$ ' denotes significant difference $(\mathrm{P}<0.05)$ as compared to W'T plants.
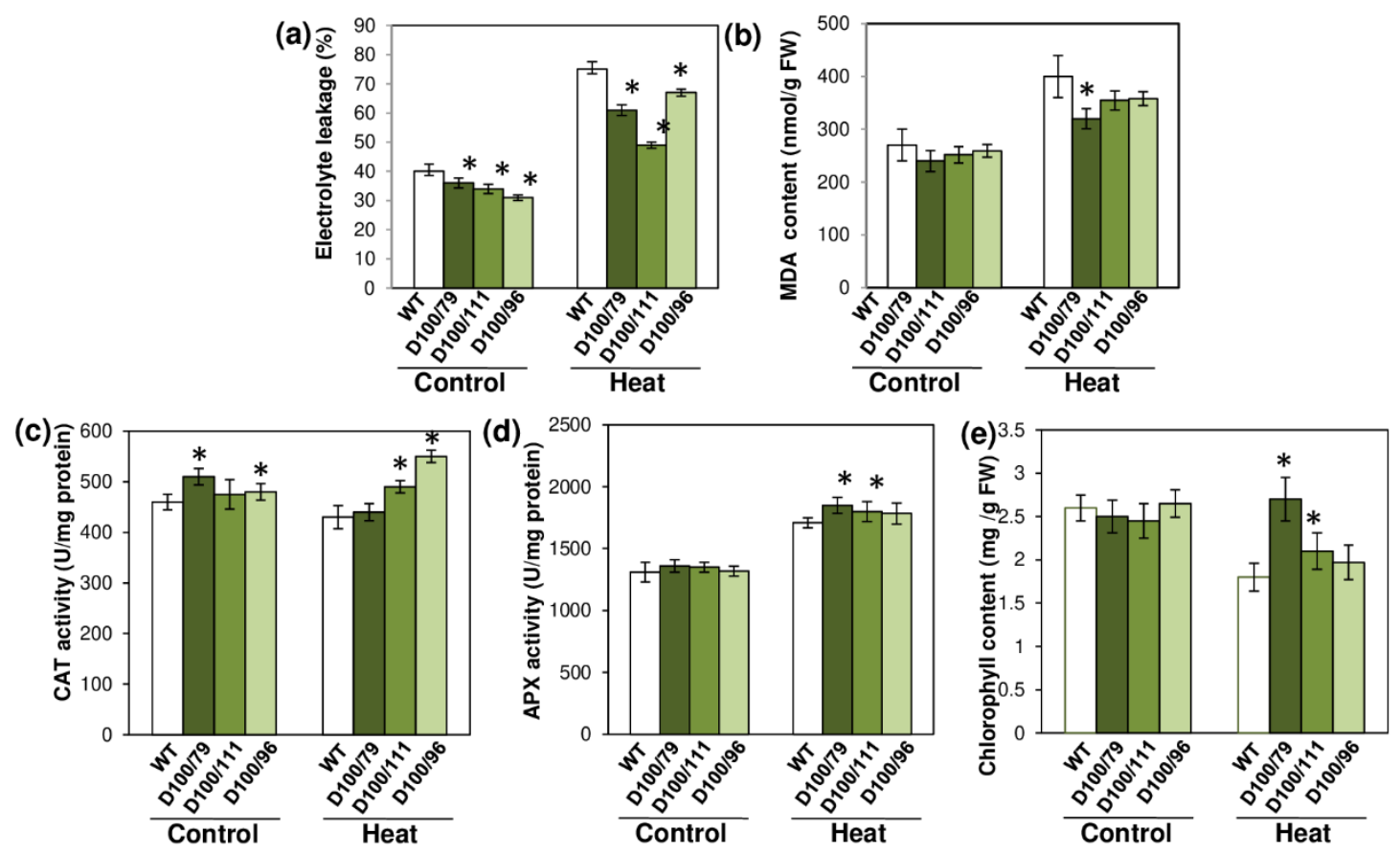

Fig 2. Analysis of physiological and biochemical parameters in WT and three selected heat-tolerant mutant $\left(\mathrm{M}_{3}\right)$ families under control conditions and after heat stress treatment $\left(45^{\circ} \mathrm{C}\right.$ for $12 \mathrm{~h}$ ). Study of (a) electrolyte leakage, (b) MDA content, (c) catalase activity, (d) APX activity and (e) chlorophyll content in selected mutant lines in comparison to WT under control conditions as well as after heat stress treatment. Vertical column represents mean of three biological replicates $\pm \mathrm{SE}$. ' ${ }^{*}$ ' denotes significant difference $(\mathrm{P}<0.05)$ as compared to respective W'T plants. 
(a)

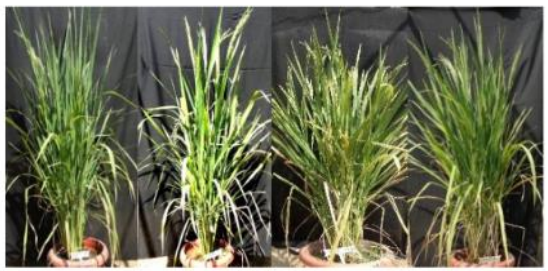

(b)

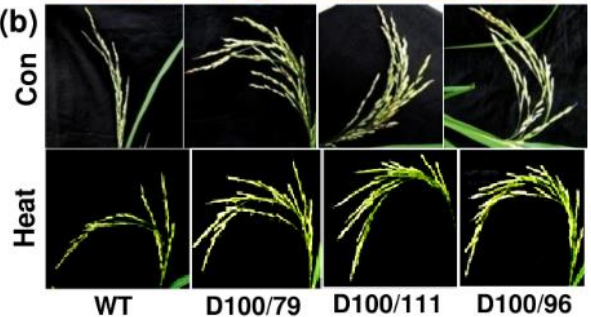

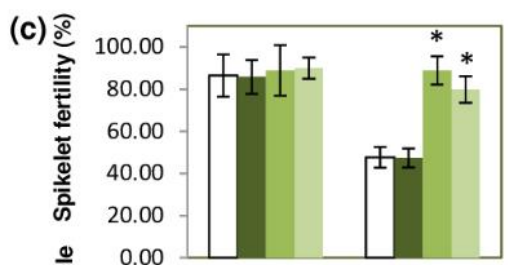

(d)

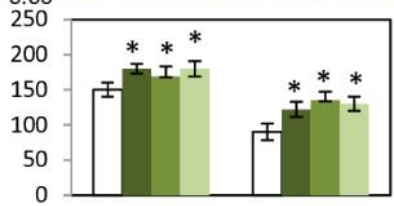

(e) 으

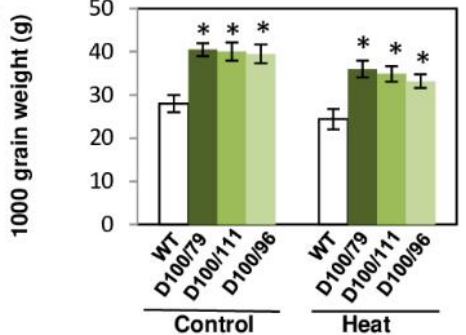

Fig 3. Analysis of morphological parameters in selected heat-tolerant mutant lines at mature (grain filling) stage after heat stress treatment $\left(40{ }^{\circ} \mathrm{C}\right.$ for $\left.4 \mathrm{~h}\right)$. At the onset of flowering, plants were transferred to a pre-set growth chamber at $40{ }^{\circ} \mathrm{C}$ for $4 \mathrm{~h}(0800 \mathrm{~h}$ to $1200 \mathrm{~h}$ ) daily for three consecutive days to allow complete flowering on the panicle from the main tiller (a) Pictorial view of WT and heat-tolerant mutants before heat stress treatment. (b) Panicle phenotype of WT and heat-tolerant mutants under control conditions (upper panel) and after heat stress treatment (lower panel). (c) Percentage of spikelet fertility, (d) number of seeds per panicle and (e) 1,000 grain weight of heat tolerant mutant lines in comparison to WT under control conditions and after heat stress treatment. Vertical column represents mean of three biological replicates \pm SE. ' $*$ ' denotes significant difference $(\mathrm{P}<0.05)$ as compared to respective WT plants.
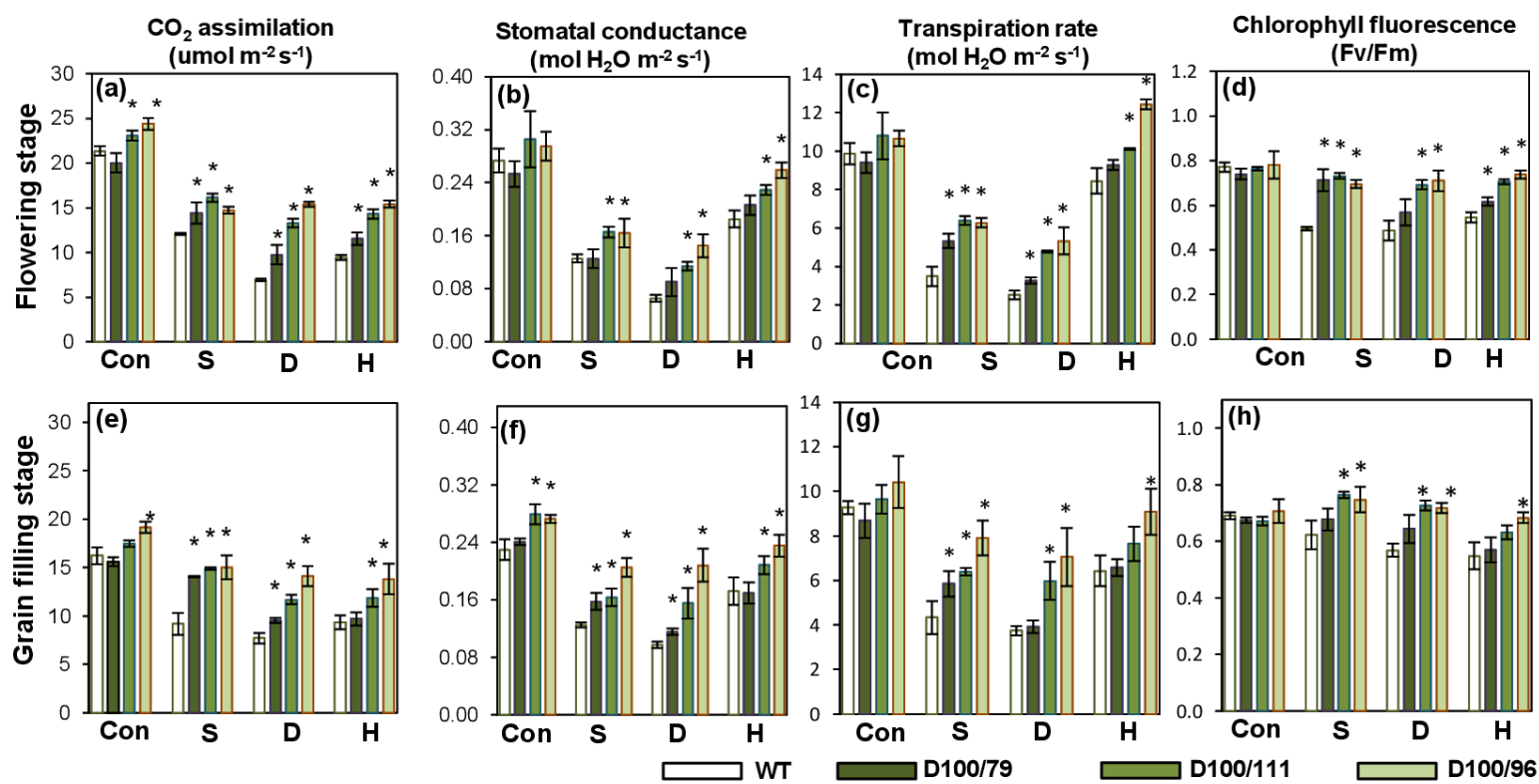

Fig 4. Analysis of gas exchange traits and chlorophyll fluorescence of WT and three selected heat tolerant mutant $\left(\mathrm{M}_{3}\right)$ families under control, drought, salinity and heat stress at anthesis (a-d) and post anthesis stage (e-h). Determination of (a) $\mathrm{CO}_{2}$ assimilation, (b) stomatal conductance, (c) transcription rate and (d) chlorophyll fluorescence in flag leaves of control, drought, salinity and heat stress treated plants at anthesis. (e), (f), (g) and (h) represent $\mathrm{CO}_{2}$ assimilation, stomatal conductance, transcription rate and chlorophyll fluorescence respectively in the flag leaves of control, drought, salinity and heat stress treated plants at the grain filling stage. Vertical column represents mean of three biological replicates $\pm \mathrm{SE}$. '*' denotes significant difference $(\mathrm{P}<0.05)$ as compared to WT plants. 


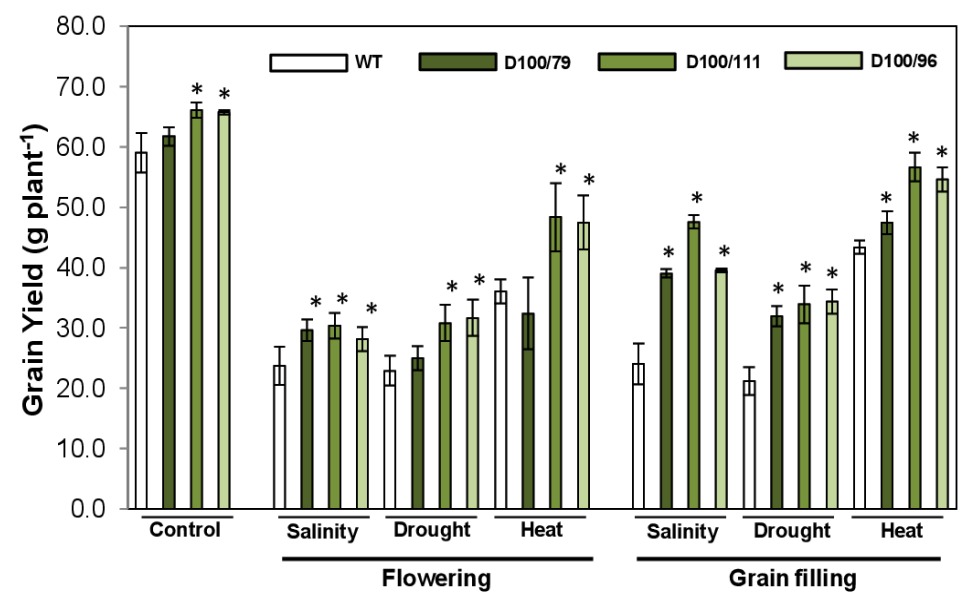

Fig 5. Grain yield per plant in WT and three selected heat-tolerant mutant $\left(\mathrm{M}_{3}\right)$ families under control, salinity, drought and heat stress at flowering and grain filling (GF) stages. Vertical column represents mean of four biological replicates \pm SE. ' $*$ ' denotes significant difference $(\mathrm{P}<0.05)$ as compared to respective control plants.

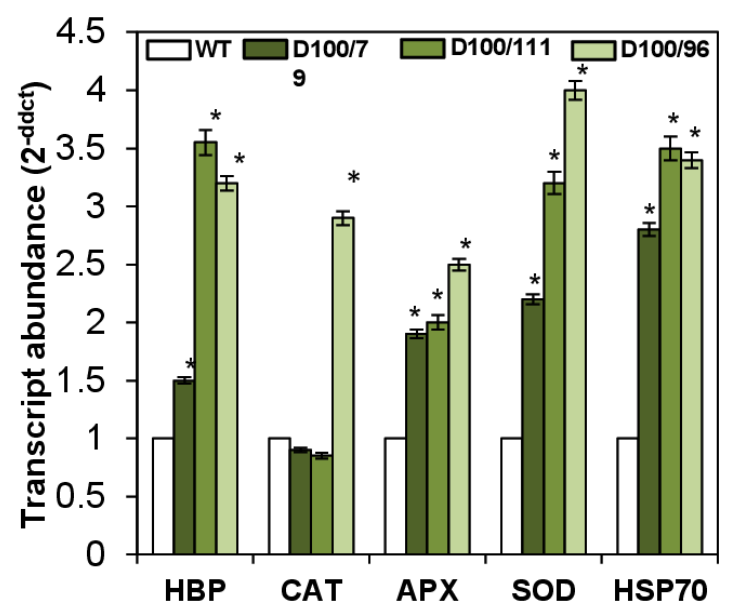

Fig 6. Molecular characterization of three selected heat-tolerant mutant $\left(\mathrm{M}_{3}\right)$ families through expression analysis of stress responsive transcripts. (a) Transcript abundance (fold change) has been shown for histone-gene binding protein (HBP), catalase (CAT), ascorbate peroxidase (APX), superoxide dismutase (SOD) and heat shock protein-70 (HSP70) in W'T and heat-tolerant mutant lines in unstressed conditions. Vertical column represents mean of three biological replicates $\pm \mathrm{SE}$. ' $*$ ' denotes significant difference $(\mathrm{P}<0.05)$ as compared to respective W'T plants.

In the present study, the selected mutant family (D100/79, D100/111 and D100/96) seedlings were found to possess better membrane stability as evident from lower electrolyte leakage, lower MDA levels, higher CAT and APX activity, and higher chlorophyll content that WT seedlings after heat stress treatment, which confirmed the lines to be superior than WT for tolerance towards high temperature (Fig 2). The three putative tolerant mutant lines were thus taken for a second round of testing for osmotic stress tolerance and characterization, where important morphological, physiological, biochemical and molecular parameters were taken into account.

Although rice is affected by high temperature at almost all growth stages (Pareek et al., 1995; Shah et al., 2011), the vegetative stage is relatively more tolerant to high temperatures. Temperatures beyond optimum thresholds reduce plant growth, alter flowering time and reduce yield (Yoshida et al., 1981). Stress coinciding with flowering and grain filing is more detrimental for grain yield (Wassmann et al., 2009; Masuduzzaman et al., 2016). Interestingly, high tolerance levels of selected mutant families to heat $\left(45^{\circ} \mathrm{C}\right)$ at the seedling stage were consistent over the later phenological stages and we observed better plant phenotype, higher spikelet fertility $(\sim 80 \%)$, increased number of spikelets per panicle and higher grain weight in the selected mutant lines (D100/79, D100/111 and D100/96) compared to wild type plants after heat stress treatment were given at the flowering or early grain filling stage (Fig 3).

Abiotic stresses are inter-connected in the agricultural environment (Pareek et al., 2010; Rao et al., 2016; Lakra et al., 2015; Faralli et al., 2015). High temperature, salinity and drought are stresses which, besides having their own unique dimensions, share osmotic stress as a common denominator (Kissoudis et al., 2014). It is important to note that plants face more than one stress at a time or during the life cycle (Tester and Bacic, 2005; Mittler, 2006; Barnabas et al., 2007). Thus, tolerance to multiple abiotic stress is needed and is the most suitable approach to tailor climate resilient rice cultivars. Owing to the impressive progress of omics-based technologies, commonality in these stresses at biochemical and molecular level has been established (Soni et al., 2015; Das et al., 2015b). Cross talk in signalling machineries has further established a strong inter-relationship between these abiotic stresses (Kissoudis et al., 2014; Das et al., 2015a). Thus, we were prompted to test the tolerance of these heat tolerant mutants for salinity and drought stress. Selected mutant 
lines recorded significantly higher $\mathrm{CO}_{2}$ assimilation rate, better transpiration rate, higher stomatal conductance and higher chlorophyll fluorescence across all three stress conditions applied at flowering or grain filling stages (Fig4). Higher photosynthetic ability and maintenance of higher levels of stomatal conductance and transpiration rates represent simple methods of stress avoidance or escape routes.

It is evident that better growth rates and higher rates of photosynthesis under stress conditions could contribute to higher yield (Long et al., 2006; Makino et al., 2011). Significantly higher grain yields were found in all three mutant families (D100/79, D100/111 and D100/96) across the stress treatments (Fig 5). In general, higher tolerance levels have a cost (yield penalty) under non-stress conditions possibly due to shifts in metabolic activity towards defence related processes. Breeders are consistently putting their efforts into improving genotypes in a way that they should provide reasonable yields under stress but should be able to produce 'at par' under non-stress conditions. Interestingly, two of the selected mutant families (D100/111, D100/96) were able to produce high yields, both under optimum as well as stress conditions. This is an interesting finding and suggests that the underlying mechanisms are constitutive. This has also been found by Bado et al. (2016b) where mechanisms for salt tolerance in rice were found to be active in both control and salt-stressed treatments. Consequently, salt-tolerance may be screened for under normal conditions.

To investigate the mechanisms of improved stress tolerance in these mutant lines, we analyzed transcript abundance of key stress-responsive genes such as, HSP 70 and HBP. HSP 70 acts as a cellular thermometer and its over-expression is correlated with improved tolerance to heat stress (Park et al., 2015). Transcript abundance analysis for HSP 70 in WT and mutants clearly demonstrated $>3$-fold higher transcript in the mutants as compared to WT seedlings under non-stress conditions. Similarly, HBP is another protein, which results in higher stress tolerance upon over-expression in tobacco (Lakra et al., 2015). The qRT-PCR based expression analysis of HBP in WT and mutants revealed higher transcript abundance in the mutants, than in WT seedlings. Transcripts corresponding to antioxidant enzymes such as, CAT, APX and SOD were also found to behave in a similar fashion where higher constitutive expression was seen in the mutants as compared to WT plants (Fig6a). However, CAT expression was found to be altered in only one mutant family i.e. D100/96 thereby suggesting multiple pathways/mechanisms operative in these mutants which may contribute towards the observed tolerance. This is in corroboration with many previous findings where abiotic stress response has shown to be highly complex and a multigenic trait (Kumari et al., 2009; Das et al., 2015b; Soda et al. 2016). Since higher constitutive expression of a representative sample of key stress response genes was found to be the possible reason for the observed differences in stress tolerance between the $\mathrm{W}^{\mathrm{T}} \mathrm{T}$ and mutant plants. Similar mechanism of stress tolerance has been shown recently to operate in natural land races of rice (Lakra et al., 2017).

From these results, we propose that these 'mutants resulted from higher constitutive expression of the representative sample (and possibly others) of key stress responsive genes and proteins, which may, in turn, be a result of deletion or modification of some unknown inhibitors of these genes due to exposure to gamma radiations. However, the findings are in good corroboration with a host of reports where differential accumulation of transcripts and proteins between contrasting genotypes has been reported for these genes (Kumari et al., 2009) and constitutive higher abundance of these genes/proteins has been reported in the tolerant genotype (Nutan et al., 2016; Soda et al., 2013; Karan et al., 2009; Bado et al., 2016b; Lakra et al 2017). Hence, we propose that the well preparedness of the mutants, to handle the stress in anticipation, seems to be a key mechanism of tolerance. Nonetheless, these mutant families will serve as useful germplasm for areas affected by a combination of stresses and the detailed molecular basis of tolerance for those mutants is yet to be revealed.

\section{Materials and Methods}

\section{Plant materials, growth conditions and stress treatments}

Rice (cv. IR64) seeds were subjected to $100 \mathrm{~Gy}$ of ${ }^{60} \mathrm{Co}$ gamma dose in a gamma chamber (BARC, India) to obtain $\mathrm{M}_{1}$ mutant seed. These were germinated and surviving $\mathrm{M}_{1}$ mutant plants $(2,000)$ were advanced by selfing to produce $\mathrm{M}_{2}$ and then $\mathrm{M}_{3}$ generations. Selection of the $\mathrm{M}_{3}$ heat-tolerant mutant families (D100/7, D100/96 and D100/111) was done by phenotypic, biochemical and molecular analyses of the plants at seedling as well as reproductive stages.

To optimise the heat stress treatment and recovery at the seedling stage, seven-day-old wild type (WT) seedlings grown under optimum growth conditions ( $12 \mathrm{~h}$ day/night, $28{ }^{\circ} \mathrm{C}, 10,000 \mathrm{Lux}$ light and $70 \%$ humidity) were subjected to high temperature (45 ${ }^{\circ} \mathrm{C}$ for 8,10 or $12 \mathrm{~h}$ ) in a growth chamber (Daihan Labtech Co. Ltd, India), followed by different durations of recovery (40, 60 or $72 \mathrm{~h}$ ) under optimum growth conditions. Based on these results, seven-day old seedlings were subjected to $12 \mathrm{~h}$ of heat shock at $45{ }^{\circ} \mathrm{C}$ followed by a 72 -h recovery period at normal temperature. Under these conditions, $50 \%$ of WT seedlings failed to recover $\left(\mathrm{LD}_{50}\right.$ for $\left.\mathrm{WT}\right)$. Following the stress treatment and recovery, leaf tissues from each line were taken for detailed experimental analysis.

Similarly, for heat stress at the flowering stage, 20 days old mutant seedlings were first transferred from hydroponic to soilfilled pots and maintained ibbenign growth conditions in a greenhouse. At the onset of flowering, plants were transferred to a pre-set growth chamber at $40{ }^{\circ} \mathrm{C}$ for $4 \mathrm{~h}(0800 \mathrm{~h}$ to $1200 \mathrm{~h})$ daily for three consecutive days to allow complete flowering on the panicle from the main tiller. As the rice inflorescence is very sensitive to high temperature (Jagadish et al., 2007), the temperature and duration of stress was reduced at the flowering stage, as compared to the seedling stage to avoid any severe effect on reproductive structures and seed set. Immediately after heat stress, the plants were transferred to recovery conditions $\left(28^{\circ} \mathrm{C}\right.$ for $72 \mathrm{~h}$ ). Following recovery, physiological and biochemical analyses were carried out. Similar to high temperature stress, salinity and drought stresses were imposed at flowering and early grain filling stages using independent sets of plants from the $\mathrm{M}_{3}$ families. For the salinity treatment, the soil of the potted mutant plants was saturated with half-strength Yoshida solution (Yoshida et al., 1981) supplemented with $200 \mathrm{mM} \mathrm{NaCl}$ for seven days and subsequently allowed to recover by watering with halfstrength Yoshida solution. For drought, responses of mutant plants were observed by water-withdrawal from the respective pots at the onset of flowering or at the early grain filling stage, the soil was allowed to dry until the soil-tensiometer reading reached 70-80 $\mathrm{kPa}$. Plants were then re-watered with halfstrength Yoshida solution for recovery. In parallel, WT plants were grown without any stress and served as control.

\section{Phenotypic and yield component analysis}

Plant phenotyping (such as root length, shoot length and fresh weight) was carried out at the seedling stage while yield component analysis (such as number of tillers/plants, spikelet fertility, grain weight and grain yield/plant) was done using data from mature plants.

\section{Electrolyte leakage}

Analysis of electrolyte leakage was carried out following the protocol of Bajji et al. (2002). Leaf samples from control and heat-treated mutant seedlings were harvested and washed with distilled water to remove any surface adhering ions. $100 \mathrm{mg}$ tissue 
was immediately dipped into $20 \mathrm{ml}$ of de-ionized water. After incubating the leaf tissues at $37^{\circ} \mathrm{C}$ for $2 \mathrm{~h}$, the electrical conductivity $\left(\mathrm{E}_{1}\right)$ of the immersion solution was measured using a conductivity meter (ELEINS, Inc., India). To determine the total conductivity $\left(\mathrm{E}_{2}\right)$, samples with immersion solution (effusate) were autoclaved for $15 \mathrm{~min}$ at $121^{\circ} \mathrm{C}$ and the conductivity of the effusate was measured after cooling to room temperature. Relative electrical conductivity was measured by the following formula: percentage of electrolyte leakage $=E_{1} / E_{2} \times$ 100 .

\section{Lipid peroxidation assay}

Lipid peroxidation was estimated by measuring the formation of malondialdehyde (MDA). MDA content was quantified by thiobarbituric acid reactive substances assay (Heath and Packer, 1968). For this purpose, about $100 \mathrm{mg}$ of leaf tissue from the control and heat-treated seedlings was homogenized in $5 \mathrm{ml}$ of $5 \% \quad(\mathrm{w} / \mathrm{v})$ trichloroaceticacid and the homogenate was centrifuged at $12,000 \times \mathrm{g}$ for 10 minat room temperature. The supernatant was mixed with an equal volume of thiobarbituric acid $[0.5 \%$ in $20 \%(\mathrm{w} / \mathrm{v})$ trichloroaceticacid] and the mixture boiled for $25 \mathrm{~min}$ at $100^{\circ} \mathrm{C}$, followed by centrifugation for $5 \mathrm{~min}$ at $7500 \mathrm{~g}$. Absorbance of the supernatant was measured at 532nm. MDA equivalents were calculated using the extinction coefficient of $155 \mathrm{mM}^{-1} \mathrm{~cm}^{-1}$.

\section{Measurement of total proteins}

Total soluble proteins were extracted from the leaves (100mg) of control and heat-treated seedlings using Zivy's buffer (Zivy et al.,1983). Amount of soluble proteins was estimated by Bradford'sassay (1976) by reading from a standard curve prepared by using different concentrations of Bovine Serum Albumin.

\section{Antioxidant enzyme activity assay}

About $100 \mathrm{mg}$ leaf material from control and heat-treated seedlings were homogenized in ice-cold $50 \mathrm{mM} \mathrm{K}_{2} \mathrm{PO}_{4}$ buffer ( $\mathrm{pH}$ 7.5) containing $2 \mathrm{mM}$ ethylenediaminetetraacetic acid (EDTA) and $0.1 \mathrm{mM}$ phenylmethylsulphonylfluoride (PMSF) The homogenizing buffer for ascorbate peroxidase (APX) additionally contained $2 \mathrm{mM}$ of $\mathrm{Na}$-ascorbate. The homogenate was centrifuged at $12,000 \times \mathrm{g}$ for $10 \mathrm{~min}$ at $4{ }^{\circ} \mathrm{C}$ and the supernatant was used for enzyme assay. Total protein content in supernatant was determined following Bradford (1976). APX activity was measured following Nakano and Asada (1981). The reaction mixture in a total volume of $1 \mathrm{ml}$ consisted of $50 \mathrm{mM}$ (pH 7.5) $\mathrm{K}_{2} \mathrm{PO}_{4}$ buffer, 0.1mM EDTA, $0.25 \mathrm{mM}$ ascorbate, 10 $\mathrm{mM} \mathrm{H} \mathrm{H}_{2} \mathrm{O}_{2}$ and enzyme extract. $\mathrm{H}_{2} \mathrm{O}_{2}$-dependent oxidation of ascorbate was followed spectrophotometrically by recording the decrease in absorbance at $290 \mathrm{~nm}$. Slope value of absorbance in $290 \mathrm{~nm}$ was considered for rate calculation. Catalase (CAT) activity was measured following the method described by Aebi (1984), by measuring the decrease in absorbance at $240 \mathrm{~nm}$ due to decomposition of $\mathrm{H}_{2} \mathrm{O}_{2}$. The slope value of the rapid decline in $240 \mathrm{~nm}$ absorption was considered for rate calculation. The reaction mixture in $1 \mathrm{ml}$ contained $50 \mathrm{mM} \mathrm{K}_{2} \mathrm{PO}_{4}$ buffer $(\mathrm{pH} 7.0)$ with leaf extract equivalent to $5 \mu \mathrm{g}$ total protein. The reaction was initiated by adding $\mathrm{H}_{2} \mathrm{O}_{2}$ to a final concentration of $20 \mathrm{mM}$.

\section{Analysis of photosynthesis parameters}

Chlorophyll fluorescence, photosynthesis $\left(\mu \mathrm{mol} \mathrm{CO} \mathrm{Cm}^{-2} \mathrm{~s}^{-1}\right)$, leaf stomatal conductance $\left(\mathrm{mol} \mathrm{H}_{2} \mathrm{O} \mathrm{m}^{-2} \mathrm{~s}^{-1}\right)$ and transpiration rate $\left(\mathrm{mmol} \mathrm{H}_{2} \mathrm{O} \mathrm{m}^{-2} \mathrm{~s}^{-1}\right)$ of the flag leaf were monitored using an InfraRed Gas Analyser (IRGA, LICOR-6400XT). Chlorophyll $a$ fluorescence and photosynthesis of the leaves of stress-treated or untreated plants (both W'T and mutant) were monitored at $25{ }^{\circ} \mathrm{C}$ under supplied $\mathrm{CO}_{2}$ concentration (400 $\mathrm{ppm})$. Leaves were dark adapted for $30 \mathrm{~min}$ prior to measurement of chlorophyll fluorescence. The minimal level of dark fluorescence $\left(\mathrm{F}_{0}\right)$ was measured under weak modulated light and the maximal intensity of fluorescence $\left(\mathrm{F}_{\mathrm{m}}\right)$ was evoked by application of a short saturating light pulse $\left(10,000 \mu \mathrm{mol} \mathrm{m}^{-2} \mathrm{~s}^{-1}\right)$. The maximal steady-state photochemical efficiency (i.e., intrinsic quantum yield under dark adapted condition) was indicated by $\left(F_{v} / F_{m}\right)$, where $F_{v}=F_{m}-F_{0}$.

\section{Quantitative real-time-PCR ( $q R T-P C R)$ analysis}

Total RNA was isolated from the flag leaf of mature (at flowering stage) plants using Tri-Reagent (Sigma-Aldrich). Elimination of genomic DNA contamination was carried out by addition of DNase I (Epicentre), as per the manufacturer's instructions. RNA samples were reverse transcribed using first strand cDNA synthesis kit (Fermentas, United States). Real-time PCR was performed using Power SYBR Green PCR Master Mix (ABI) on ABI prism 7900 Real-Time PCR system. Primer pairs (Table 1) for transcript expression analysis of stress responsive genes was designed using ABI primer designer. Actin was used as the reference gene in the expression analysis. The $2^{-\mathrm{ddCT}}$ method was used to analyse the fold change in transcript expression (Livak and Schmittgen, 2001).

\section{Statistical analysis}

Physiological and biochemical experimental analyses were repeated three times using three independent mutant plants from each mutant family. All the experiments were analysed as a completely randomized design (CRD) with two-way ANOVA. Means were compared using least significant difference (LSD) at $\mathrm{P}<0.05$.

\section{Conclusions}

Three selected mutant $\mathrm{M}_{3}$ families (D100/79, D100/111 and D100/96) were found to have higher transcript and protein abundance, better plant phenotype, better antioxidant response, higher spikelet fertility, higher photosynthetic activity and higher grain yield under heat, salinity and drought stress as compared to the wild type parental line. It has been confirmed from the study that gamma irradiation could create useful mutations in the rice genome which could ultimately enhance tolerance to multiple abiotic stress conditions. The region(s) of mutation in these novel lines is yet to be discovered which will further open inroads of molecular basis of stress tolerance. The selected mutant lines provide useful germplasm for use in breeding programmes for abiotic stress tolerance in rice.

\section{Acknowledgements}

Authors are thankful to International Atomic Energy Agency (IAEA) for financial support to carry out the work. Research in the laboratory of AP is also supported from funds received from Ministry of Science and Technology, India and Resource Network Program from University Grants Commission (UGC-RNW) through the University. PD is thankful to University Grants Commission (UGC) for Dr D. S. Kothari Fellowship awarded to her.

\section{References}

Aebi H (1983) Catalase. In: Bergmeyer HU (ed) Methods of Enzymatic Analysis, Academic Press, Inc, New York, NY, USA. 273-288.

Ahloowalia BS, Maluszynski M, Nichterlein K (2003) Global impact of mutation-derived varieties. Euphytica. 135: 187-204.

Ayeneh A, Van Ginkel M, Reynolds MP, Ammar K (2002) Comparison of leaf, spike, peduncle and canopy temperature depression in wheat under heat stress. Field Crops Res. 79: 173-184. 
Bado S, Forster BP, Ghanim AMA, Jankowicz-Cieslak J, Berthold G, Gregorio GB, Luxiang L (2016a) Protocols for pre-Field screening of mutants for salt tolerance in rice, wheat and barley. Springer Open Access, ISBN 978-3-319-26588-9.

Bado S, Forster BP, Nielen S, Ali AM, Lagoda PJL, Laimer M (2015) Plant Mutation Breeding: Current Progress and Future Assessment. In: Janick J (ed) Plant Breeding Reviews,39(Chapter 2): 23-88.

Bado S, Forster BP, Padilla-Alvarez R, Resch C, Migliori A, Diawara Y, Jaksic M and Laimer M (2016) Prediction of salt tolerance in rice (Oryza sativa) based on shoot element content in non-stressed conditions. J Mater Sci Eng. A6: 1-16.

Bahuguna RN, Jha J, Pal M, Shah D, Lawas LM, Khetarpal S, Jagadish KS (2015) Physiological and biochemical characterization of NERICA-L-44: a novel source of heat tolerance at the vegetative and reproductive stages in rice. Physiol plant. 154(4): 543-559

Bajji M, Kinet JM, Lutts S (2002) The use of the electrolyte leakage method for assessing cell membrane stability as a water stress tolerance test in durum wheat. Plant Growth Regul. 36: 61-70.

Baloch AW, Soomro AM, Javed MA, Bughio HR, Alam SM, Bughio MS, Mohammed T, Mastoi NN (2003) Induction of salt tolerance in rice through mutation breeding. Asian J Plant Sci. 2: 273-276.

Barnabas B, Jager K, Feher A (2008) The effect of drought and heat stress on reproductive processes in cereals. Plant cell environ. 31(1): 11-38.

Bradford MM (1976) A dye binding assay for protein. Annals Biochem. 72: 248-54.

Cassells AC, Doyel BM (2003) Genetic engineering and mutation breeding for tolerance to abiotic and biotic stresses: science, technology and safety. Bulgarian J Plant Physiol (Special Issue). $52-82$.

Chhun T, Taketa S, Tsurumi S, Ichii Z (2003) Interaction between two auxin-resistant mutants and their effects on lateral root formation in rice (Oryza sativa L.). J Exp Bot. 54: 27012708.

Das P, Mishra M, Lakra N, Singla-Pareek SL, Pareek A (2014) Mutation breeding: a powerful approach for obtaining abiotic stress tolerant crops and upgrading food security for human nutrition. In: Tomlekova N, Kojgar I, Wani R (eds) Mutagenesis: Exploring Novel Genes and Pathways, Wageningen Academic Publisher, Wageningen. 15-36.

Das P, Nutan KK, Singla-Pareek SL, Pareek A (2015a) Oxidative environment and redox homeostasis in plants: dissecting out significant contribution of major cellular organelles. Front Environ Sci.2: 70.

Das P, Nutan KK, Singla-Pareek SL, Pareek A (2015b) Understanding salinity responses and adopting 'omics-based' approaches to generate salinity tolerant cultivars of rice. Front Plant Sci. 6: 712.

Faralli M, Lektemur C, Rosellini D, Gürel F (2015) Effects of heat shock on salinity tolerance in barley (Hordeum vulgare L.): plant growth and stress-related gene transcription. Biol Plant. 59(3):537-546.

Grover A, Minhas D (2000) Towards production of abiotic stress tolerant transgenic rice plants: issues, progress and future research needs. Proc IndianNatl Sci Acad, Part B. 66(1): 1332

Hayashi Y, Takehisa H, Kazama Y, Kanba C, Ohbu S, Ryuto H, Fukunishi N, Tokairin H, Ohkoshi K, Yoshini Y, Sato T, Abe $\mathrm{T}$ (2008) Characterization of salt tolerant mutants of rice induced by heavy ion irradiation. RIKEN Accelerator Progress Report. 41: 234.

Hayashi Y, Takehisa H, Kazama Y, Ichida H, Ryuto H, Fukunishi N, Abe T, Kamba C Sato T (2007). Effects of ion beam irradiation on mutation induction in rice. Paper presented in Eighteenth International Conference, Cyclotrons and Their Applications. Catania, Italy, 1-5 October 2007, 1-5.

Heath RL, Packer L (1968) Photoperoxidation in isolated chloroplasts: I. Stoichiometry of fatty acid peroxidation. Arch Biochem Biophys. 125: 189-98.

Hoagland DR, Arnon DI (1938) The water-culture method for growing plants without soil. Circular. California Agricultural Experiment Station. 347: 32.

Jagadish SVK, Craufurd PQ, Wheeler TR (2007) High temperature stress and spikelet fertility in rice (Oryzasativa L.). J Exp Bot. 58: 1627-1635.

Jain MS, Suprasanna P, (2011) Induced mutations for enhancing nutrition and food production. Gene Conserve. 40: 201-215.

Joshi R, Prashat R, Sharma PC, Singla-Pareek SL, Pareek A (2016) Physiological characterization of gamma-ray induced mutant population of rice to facilitate biomass and yield improvement under salinity stress. Indian J Plant Physiol. 21:545-555.

Karan R, Singla-Pareek SL, Pareek A (2009) Histidine kinase and response regulator genes as they relate to salinity tolerance in rice. Funct Integr Genomics. 3: 411-417.

Kissoudis C, van de Wiel C, Visser RGF, Van Der Linden G (2014) Enhancing crop resilience to combined abiotic and biotic stress through the dissection of physiological and molecular crosstalk. Front Plant Sci. 5: 207.

Kumari S, Panjabi nee Sabharwal V, Kushwaha HR, Sopory SK, Singla-Pareek SL, Pareek A (2009) Transcriptome map for seedling stage specific salinity stress response indicates a specific set of genes as candidate for saline tolerance in Oryza sativa L. Funct Integr Genomics. 9(1): 109-123.

Laborte AG, Paguirigan NC, Moya PF, Nelson A, Sparks AH, Gregorio GB (2015) Farmers' preference for rice traits: Insights from farm surveys in central luzon, Philippines. Plos One. 10: 1966-2012.

Lakra N, Kaur C, Anwar K, Singla-Pareek SL, Pareek A (2017) Proteomics of Contrasting Rice Genotypes: Identification of Potential Targets for Raising Crops for Saline Environment. Plant Cell Environ. DOI: 10.1111/pce.12946.

Lakra N, Nutan KK, Das P, Anwar K, Singla-Pareek SL, PareekA (2015) A nuclear-localized histone-gene binding protein from rice (OsHBP1b) functions in salinity and drought stress tolerance by maintaining chlorophyll content and improving the antioxidant machinery. J plant physiol: 176:3646.

Livak KJ, Schmittgen TD (2001) Analysis of relative gene expression data using real-time quantitative PCR and the 2[-Delta Delta C(t)] method. Methods. 25: 402-8.

Lobell DB, Gourdji S(2012) The influence of climate change on global crop productivity. Plant Physiol. 160: 1686-1697.

Long SP, ZhuXG, Naidu SL, Ort DR (2006) Can improvement in photosynthesis increase crop yields? Plant Cell Environ. 29: 315-330.

Makino A, (2011) Photosynthesis, grain yield, and nitrogen utilization in rice and wheat. Plant physiol. 155(1): 125-129.

Masuduzzaman ASM, Ahmad HU, Haque M, Ahmed MME (2016) Evaluation of rice lines tolerance to heat during their flowering stage. Rice Research-Open Access. 4: 170.

Mittler R (2006) Abiotic stress, the field environment and stress combination. Trends Plant Sci. 11: 15-19.

Nakano Y, Asada K (1981) Hydrogen peroxide is scavenged by ascorbate-specific peroxidase in spinach chloroplasts. Plant Cell Physiol. 22: 867-80.

Nguyen NV, Ferrero A (2006) meeting the challenges of global rice production. Paddy Water environ. 4: 1-9.

NutanKK, Kushwaha HR, Singla-Pareek SL, Pareek A (2017) Transcription dynamics of Saltol QTL localized genes encoding transcription factors, reveals their differential regulation in contrasting genotypes of rice. Funct Integr Genomics.17(1): 69-83 
Pareek A, Singla-Pareek SL, Grover A (1995) Immunological evidence for accumulation of two high molecular weight (104 and $90 \mathrm{kDa}$ ) HSPs in response to different stresses in rice and in response to high temperature stress in diverse plant genera. Plant Mol Biol. 29: 293-301.

Pareek A, Singla-Pareek SL, GroverA (1997) Short term salinity and high temperature stress-associated ultrastructural alterations in young leave cells of Oryza sativa L. Annals Bot. 80: 629-639.

Pareek A, Sopory SK, BohnertHJ, Govindjee (2010) Abiotic Stress Adaptation in Plants: Physiolgical, Molecular and Genomic Foundation. Publisher: Springer, The Netherlands.

Park HJ, Jung WY, Lee SS, Song JH, Kwon S-Y, Kim HR, Kim CW, Ahn JC, Cho HS (2013) Use of heat stress responsive gene expression levels for early selection of heat tolerant cabbage (Brassica oleraceaL.) International JMol Sci. 14: 1187111894

Parry MAJ, Madgwick PJ, Bayon C, Tearall k, Lopez AH, Baudo M, Rakszegi M, Hamada W, Al-Yassin A, Ouabbou H, Labhilili M, Phillips AL (2009) Mutation discovery for crop improvement. JExp Bot. 60:2817-2825.

Sakata T, HigashitaniA (2008) Male sterility accompanied with abnormal anther development in plants-genes and environmental stresses with special reference to high temperature injury. Int J Plant Dev Biol. 2: 42-51.

Saleem MY, Mukhtar Z, Cheema AA, Atta BM (2005) Induced mutation and in vitro techniques as a method to induce salt tolerance in Basmati rice (Oryza sativa L.). Int J environ Sci Technol. 2: 141-145.

Shah F, Huang J, Cui K, Nie L, Shah T, Chen C, Wang K (2011) Impact of high-temperature stress on rice plant and its traits related to tolerance. J Agr Sci. 149: 545-556.

SinghBD (2000) Plant Breeding-Principles and Methods. Kalyani Publisher. Narosa Publishing House. India.

Soda N, Kushwaha HR, Soni P, Singla-Pareek SL, Pareek A (2013) A suite of new genes defining salinity stress tolerance in seedlings of contrasting rice genotypes. Funct Integr Genomics. 13: 351-365.
Soda N, SharanA, Gupta BK, Singla-Pareek SL, Pareek A (2016) Evidence for nuclear interaction of a cytoskeleton protein (OsIFL) with metallothionein and its role in salinity stress tolerance. Sci Rep.6: 34762.

Soni P, Nutan KK, Soda N, Nongpiur RC, Roy S, Singla-Pareek SL, Pareek A (2015) Towards understanding abiotic stress signaling in plants: convergence of genomics, transcriptomics, proteomics and metabolomics approaches. In: Pandey GA (ed) Elucidation of Abiotic Stress Signaling in Plants, Springer, New York, USA. 3-40

Sullivan CY (1972) Mechanisms of heat and drought resistance in grain sorghum and methods of measurement. In: Rao NGP and House LR (eds. Sorghum in The Seventies, pp. 247-264. Oxford and IPH, New Delhi, India.

Tester M, Bacic A (2005) Abiotic stress tolerance in grasses. From model plants to crop plants. Plant Physiol. 137: 791-793.

Wassmann R, Jagadish SVK, Heuer S, Ismail A, Redona E, Serraj R, Singh RK, Howell G, Pathak H, Sumfleth K (2009) Climate Change Affecting Rice Production: The Physiological and Agronomic Basis for Possible Adaptation Strategies. In: Donald LS (ed) Advances in Agronomy, Burlington. 59-122.

Yokoi S, Quintero FJ, Cubero B, Ruiz MT, Bressan RA, Hasegawa PM, Pardo JM (2002) Differential expression and function of Arabidopsis thaliana $\mathrm{NHX} \mathrm{Na} / \mathrm{H}^{+}$antiporters in the salt stress response. Plant J. 30: 529-539.

Yoshida S, Satake T, Mackill D (1981) High temperature Stress. IRRI Research Paper. 67: 1-15.

Zivy M, Thiellement H, Vienne D, Hofmann JP (1983) Study on nuclear andcytoplasmic genomic expression in wheat by twodimensional gel electrophoresis. TheorAppl Genet. 66: 1-7. 